\title{
EXPERIMENTS CONCERNING THE SEXUAL DIFFER- ENCE IN THE WING LENGTH OF DROSOPHILA AMPELOPHILA
}

\author{
FRANK E. LUTZ \\ The American Museum of Natural History, New York \\ TWO FIGURES
}

Two of the forms of the fruit fly, Drosophila ampelophila, which have been isolated recently by Prof. T. H. Morgan are distinguished from the normal by lesser wing length. One, called 'wingless' in Professor Morgan's papers, really possesses vestiges of wings which appear to consist largely of modified basal portions of normal wings. The other, called 'miniature,' possesses all the veins of the normal wing in approximately normal condition but the wing is only about two-thirds the normal length.

'Winglessness' is recessive to normality according to the simple Mendelian formulae. In $\mathrm{F}_{1}$ all individuals, both male and female, are hybrid no matter which parent bears the abnormal character. The 'miniature' wings are also recessive but are sex limited in their inheritance. If the mother have miniature wings and the father be normal, only the females of $F_{1}$ will be hybrids while all the males will be pure recessives. In the reciprocal cross the females will again be hybrids but the males will be pure dominants. The reasonable explanation which Professor Morgan has advanced of these phenomena is that the factor for miniature wings (using such an expression in lieu of a better) is contained in, or in some way connected with, that chromosome of which the female possesses two and the male but one, while the factor for winglessness is connected with something which is shared equally by both sexes. This idea is shown diagrammatically in figure 1 in which the composition of pure stock of the three forms and that of two of the cross are shown, the $X$-chromosomes being represented by squares. 


\section{Pure Females}
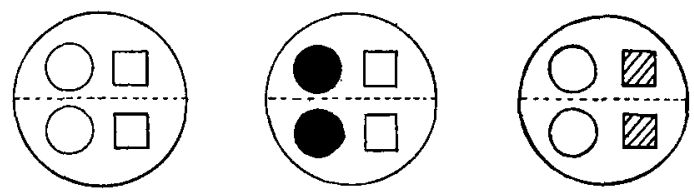

Pure Máles
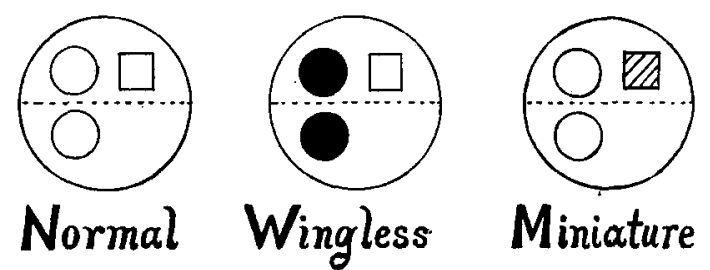

Wingless

Miniature

Normal $\times$
Wingless

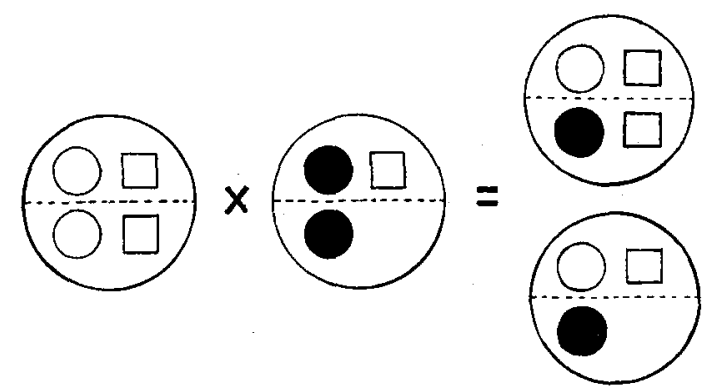

Normal $\mathrm{x}$
Miniature
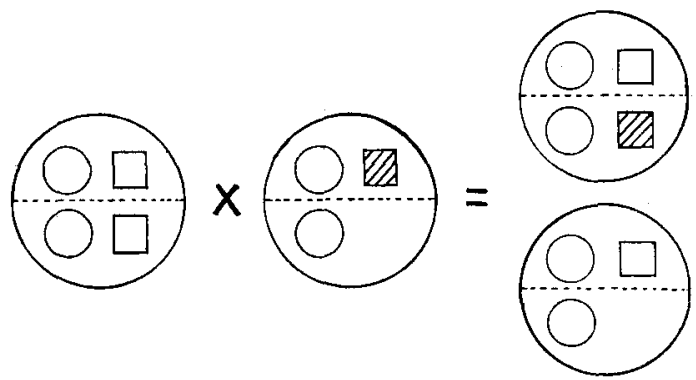

Fig. 1 Theoretical composition of zygotes. The squares represent the $X$ chromosome. The small circles represent the rest of chromosomes. Where they are plain their composition is supposed to be normal. 
TABLE 1

Average dimensions. See text.

\begin{tabular}{|c|c|c|c|c|}
\hline & & $\begin{array}{c}\underset{\text { NORMAL }}{\times} \\
\text { NORMAL } \sigma^{7}\end{array}$ & $\begin{array}{c}\underset{X}{\text { NORMAL }}+9 \\
\text { WINGLERS } \sigma^{7}\end{array}$ & 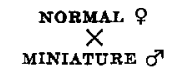 \\
\hline \multirow{2}{*}{ Wing......... } & q & $71.404 \pm 0.180$ & $65.644 \pm 0.210$ & $70.932 \pm 0.139$ \\
\hline & $\sigma^{7}$ & $64.025 \pm 0.137$ & $58.568 \pm 0.151$ & $64.737 \pm 0.149$ \\
\hline \multirow{2}{*}{ Femur........ } & ㅇ & $73.059 \pm 0.162$ & $69.695 \pm 0.236$ & $71.045 \pm 0.157$ \\
\hline & $\sigma^{x}$ & $69.194 \pm 0.170$ & $68.053 \pm 0.160$ & $69.651 \pm 0.174$ \\
\hline \multirow{3}{*}{ Wing : Femur. } & 우 & $99.199 \pm 0.128$ & $93.851 \pm 0.156$ & $98.409 \pm 0.145$ \\
\hline & $\sigma^{x}$ & $91.783 \pm 0.116$ & $85.990 \pm 0.175$ & $92.767 \pm 0.133$ \\
\hline & $q-\sigma^{7}$ & $7.416 \pm 0.173$ & $7.861 \pm 0.234$ & $5.642 \pm 0.197$ \\
\hline
\end{tabular}

It will be seen from table 1 that the mean wing length of homozygous normal females is considerably greater than that of similar males - their brothers. The average length of the middle femur is also greater in the females than in the males but the sexual dimorphism with respect to wing length is relative as well as absolute, as is shown by the fact that the ratio of wing to femur among the females greatly exceeds that among the males, the difference between the averages being nearly fifty times the error of the difference. Even so, this would not prove the existence of a fundamental sexual dimorphism if there were a tendency for generally large flies to have the ratio large. Without entering the maze of spurious correlation caused by using indices, we can see from the regression lines (fig. 2) that in both sexes, but especially in the male, there is a tendency for the wing to get proportionately smaller as the general size of the insect, as measured by the size of the middle femora, increases. The dimorphism is therefore real. The sexes are built on different plans.

It is in all ways probable that there is a large complex of factors concerned in the development of a normal wing. It is possible that the abnormal forms considered here are caused by the dropping out of certain of the factors from this complex. It would seem that the normal females get a double dose and the males but a single dose of those factors of the normal complex which are connected with the $X$-chromosomes. It is well known that many factors do not cause as great a somatic development when in a simplex condition (for example, heterozygous) as these 


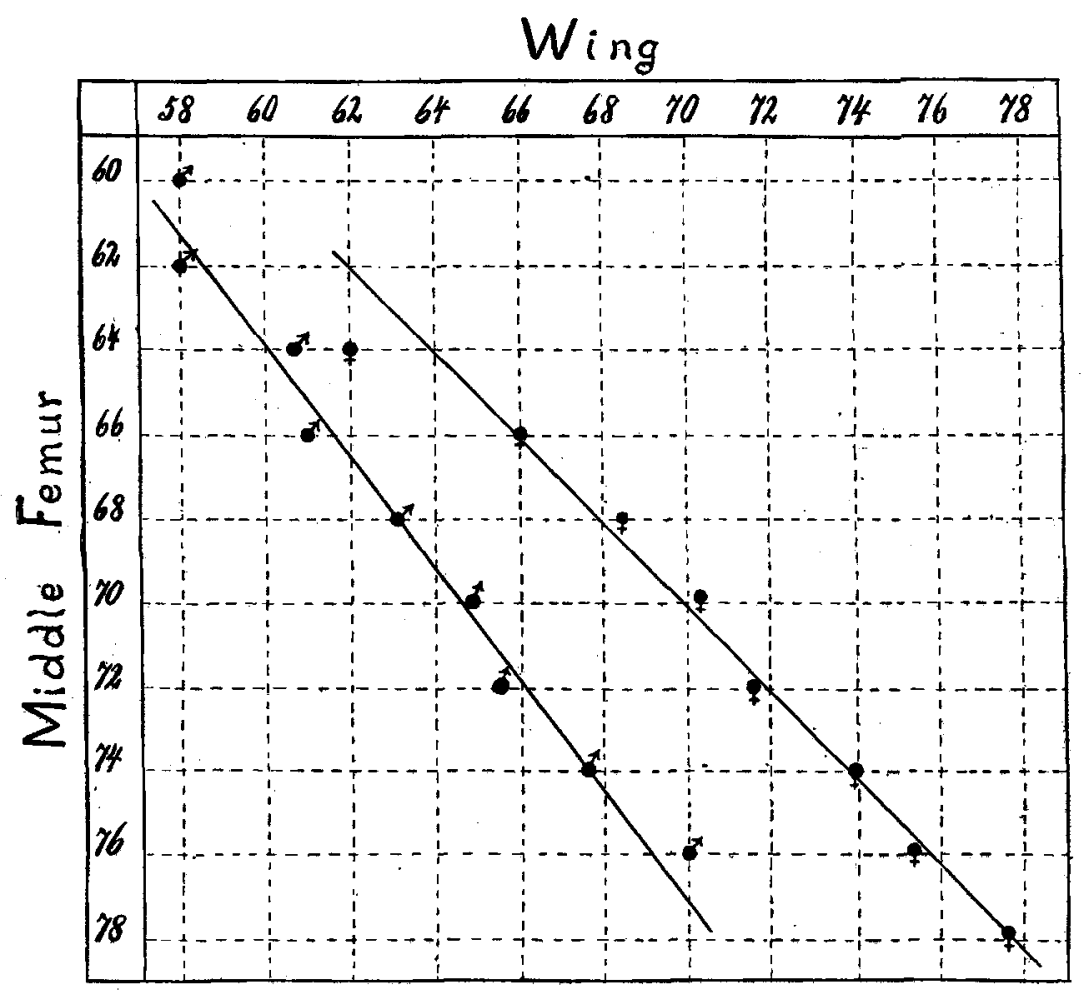

Fig. 2 Regression lines for homozygous normal flies.

same factors cause when in a duplex or homozygous condition. May it not be, then, that the greater wing development in normal females is caused by their getting a double dose of certain germinal elements, one dose for each $X$-chromosome, while the males get but a single dose? The following experiments were tried in the hope of getting some answer to this question.

All the flies in the three sets of experiments were reared at the same time, given an abundance of food from the same jar of fermenting banana, kept in the same sort of bottles which were placed side by side on the table. Furthermore, the flies for each experiment were the offspring of several score of freely interbreeding parents and were reared in a number of different bottles so that the chance variations of ancestral bias among the parents and 
of environmental effects from bottle to bottle would tend to be equalized in the three sets of offspring. This care was taken because of the marked influence environmental conditions, at least, have upon general body size.

Delcourt and Guyenot ${ }^{1}$ have suggested that in my note on the failure of disuse to decrease wing length, I should have given the ratio of wing length to body length. It did not, and still does not, seem to me to be necessary since all the factors involved, except disuse of wings, tended to decrease the general body size as much as or more than that of the wing. In this paper, however, it does seem desirable to have some other character with which to compare the wing. Among all that are feasible the body length is the worst because it may change from hour to hour in the living insect, can be measured only with great difficulty and changes greatly after death. I have used the length of the middle femora because it has none of these disadvantages. The wings and legs used here were removed from freshly etherized flies and immediately mounted in balsam. Measurements were made and are recorded in units of $\frac{1}{36} \mathrm{~mm}$. for the wings and $\frac{1}{100} \mathrm{~mm}$. for the femora.

Taking up first the offspring of normal females $x$ wingless males, we find that, while the wing is approximately of normal length in both sexes, both it and the femora are significantly smaller than in the homozygous normal material. This may possibly be an environmental effect which was not entirely avoided by the cultural methods used. However, the ratio of the wing to femur is also significantly smaller in both sexes. It is smaller even though, as was pointed out above, there is a tendency for small flies to have the ratio larger than in the case of large flies.

${ }^{1}$ Bull. Seient. France et Belgique, 7th Serie, tom. 45, no. 4. In a general denunciation of all the work hitherto done with this insect, they deplore the fact that the results have been obtained without the extreme refinements of bacteriological and physiological methods which they recommend. Their criticisms, insofar as they have any value, can be applied only to the study of fluctuating variants such as the characters considered here. All attempts to get heritable abnormal venation or such forms as wingless and miniature by purposely using extreme environmental conditions have failed. It is, therefore, absurd to lay stress in such cases upon the slight variations of environment from bottle to bottle. 
The heterozygous wings are to all appearances normal but they are really not relatively as long as normal.

As is indicated in figure 1, both sexes of these flies have only one dose of that part of the normal wing complex which presumably dropped out to give a wingless fly, whereas both sexes of normal flies have two doses. In wingless flies, and hence in half of each zygote from which these hybrids came, something has been changed, at least, so that the tendency to develop the wings to normal proportions has been lost. It is probable that the result of this experiment, a phenomenon usually referred to as 'incomplete dominance,' is due to this cause.

However that may be, the interesting point for the present discussion is that the sexual dimorphism has not been changed. Environmental effects are practically ruled out here since the males and females of a given experiment grew up together. Normal females have a wing-to-femur ratio 7.42 greater than the males and in these flies it is 7.86. The difference of 0.44 is less than twice the probable error and certainly is not significant. On the hypothesis stated above, this is what is to be expected since the germinal changes are alike in the two sexes.

Conditions are theoretically quite different in the cross between normal females and miniature-winged males. It will be seen from figure 1 that normal females get two doses of that part of the wing complex which is connected with the sex chromosome whereas the males get but one. In order that miniature wings may appear, this part of the complex must be changed, either by the dropping out of a factor or in some other way. In the cross just mentioned the male offspring are, according to theory, perfectly normal in their germinal make up. Their single $X$ chromosome has it full share, and no more, of wing factors. The female, however, has only one normal $X$-chromosome. The other either lacks a factor or, less probably, has a new modifying factor. At any rate the second $X$-chromosome is not equipped for full wing development. Hence the sexes are more nearly alike in their germinal make up than are the normal. The results show that they are also more nearly alike in their somatic condition. 
The difference between these males and the normal males with respect to the wing-to-femur ratio must be considered as an environmental effect since they are supposed to be germinally the same. It is $1.01 \pm 0.15$ and is doubtless significant in spite of the precautions taken. However, as was pointed out above, we escape even this difficulty when comparing brothers and sisters since there is no evidence and it is not believable that a given environmental condition will operate to increase the relative size of the brothers' wings and decrease that of the sisters' as is the case in this cross. The sexual difference is only 5.64, that is, $1.78 \pm 0.25$ less than normal. The sexual difference in these flies is still nearly thirty times its probable error but in the normal lot it is about fifty.

An explanation of this remaining and still considerable sexual difference is not difficult of framing on the hypothesis here followed. In fact, a wiping out of all sexual difference would have proved too much. It is only recently that any sex-limited characters have been known. It is more than likely that there are many factors concerned in wing development. Certainly all the factors have not been isolated since wings have not been entirely done away with even in the so-called wingless strain. We are free, then, to postulate that it is these remaining factors which cause the remaining sexual dimorphism of wing length.

Therefore it seems that, while proof is lacking, indications have been found that the greater wing development in the normal females of this fly than in the males is due to the females getting two sets of those wing factors which are connected with the $X$ chromosome while the males get but one, the double set causing a greater somatic effect than the single. 